\title{
Fluid shear stress inhibits vascular inflammation by decreasing thioredoxin- interacting protein in endothelial cells
}

\author{
Hideyuki Yamawaki, ${ }^{1}$ Shi Pan, ${ }^{1}$ Richard T. Lee, ${ }^{2}$ and Bradford C. Berk ${ }^{1}$ \\ ${ }^{1}$ Center for Cardiovascular Research, University of Rochester, Rochester, New York, USA. ²Cardiovascular Division, Brigham and Women's Hospital, \\ Harvard Medical School, Cambridge, Massachusetts, USA.
}

\begin{abstract}
Regions in the vasculature that are exposed to steady laminar blood flow are protected from atherosclerosis as compared with regions where flow is disturbed. We found that flow decreased TNF-mediated VCAM1 expression by inhibiting JNK and $\mathrm{p} 38$. JNK inhibition correlated with inhibition of apoptosis signal-regulating kinase 1 (ASK1), a JNK and p38 activator. Thioredoxin-interacting protein (TXNIP) is a stress-responsive protein that inhibits thioredoxin (TRX) activity. Since thioredoxin inhibits ASK1, we hypothesized that changes in TXNIP-TRX-ASK1 interactions mediate the antiinflammatory effects of flow. To explore this, we used perfused vessels and cultured ECs. Exposure of rabbit aortae or ECs to normal flow $\left(12 \mathrm{dyn} / \mathrm{cm}^{2}, 24\right.$ hours) was associated with decreased TXNIP expression and increased TRX activity compared with exposure to low flow $\left(0.4 \mathrm{dyn} / \mathrm{cm}^{2}\right)$. Normal flow inhibited TNF activation of JNK/p38 and VCAM1 expression. In cultured ECs, reduction of TXNIP expression by small interfering RNA increased TRX binding to ASK1 and inhibited TNF activation of JNK/p38 and VCAM1 expression. Conversely, overexpression of TXNIP stimulated JNK and p38. In aortae from TXNIP-deficient mice, TNF-induced VCAM1 expression was inhibited. The data suggest that TXNIP and TRX are key components of biomechanical signal transduction and establish them as potentially novel regulators of TNF signaling and inflammation in ECs.
\end{abstract}

\section{Introduction}

Substantial evidence exists that physiologic fluid shear stress exerts atheroprotective effects in vivo, since atherosclerosis preferentially occurs in areas of disturbed flow or low shear stress, whereas regions with steady laminar flow and physiologic shear stress are protected $(1,2)$. Pathogenic features of atherosclerosis are oxidative stress and inflammation characterized by endothelial expression of VCAM1 (3). We have proposed that steady laminar flow inhibits VCAM1 expression by increasing antioxidant mechanisms and blocking inflammatory signaling events. Specifically, using in vitro-cultured ECs $(4,5)$ and ex vivo intact vessels (6), we have demonstrated that one mechanism by which steady laminar flow is atheroprotective involves inhibition of TNF-mediated activation of the apoptosis signal-regulating kinase 1-JNK/p38 kinase pathway.

Apoptosis signal-regulating kinase 1 (ASK1), a mitogenactivated protein kinase kinase kinase, plays essential roles in cytokine-related signaling and stress-induced apoptosis (7). Through genetic screening for ASK1-binding proteins, Saitoh et al. (8) found that thioredoxin (TRX) bound directly to the $\mathrm{N}$-terminus of ASK1 and inhibited ASK1 kinase activity as well as ASK1-dependent apoptosis. TRX is a ubiquitous thiol oxidoreductase that regulates cellular redox status. TRX can protect against oxidative stress-induced cell injury or inflammation directly via antioxidant effects and indirectly by protein-protein

Nonstandard abbreviations used: ASK1, apoptosis signal-regulating kinase 1; BAEC, bovine aortic EC; HUVEC, human umbilical vein EC; siRNA, small interfering RNA; TRX, thioredoxin; TXNIP, thioredoxin-interacting protein.

Conflict of interest: The authors have declared that no conflict of interest exists.

Citation for this article: J. Clin. Invest. 115:733-738 (2005)

doi:10.1172/JCI200523001. interaction with signaling molecules such as ASK1 (9). TRX also exhibits growth-promoting effects presumably via an increased supply of reducing equivalents for DNA synthesis and activation of transcription factors that regulate cell growth. Thioredoxininteracting protein (TXNIP, also termed VDUP1 for vitamin $\mathrm{D}_{3}{ }^{-}$ upregulated protein 1) was originally identified in HL-60 leukemia cells treated with 1,25-dihydroxyvitamin $\mathrm{D}_{3}(10)$. Thereafter, Nishiyama et al. (11) isolated TXNIP as a TRX-binding protein using a yeast 2 -hybrid system. Biochemical analysis showed that TXNIP inhibited TRX activity by interacting with the catalytic center of TRX (cysteines 32 and 35); this suggests that TXNIP is an endogenous inhibitor of $\operatorname{TRX}(11,12)$. It seems likely that TXNIP competes with ASK1 for binding to TRX, thereby releasing ASK1 from TRX inhibition.

There is accumulating evidence that TXNIP plays a pivotal role in cardiovascular disorders, functioning as a sensor for biomechanical and oxidative stress. Schulze et al. recently reported in vascular smooth muscle that hyperglycemia increased oxidative stress by inducing TXNIP and inhibiting the antioxidant function of TRX (13). They also showed that diabetic animals exhibited increased vascular expression of TXNIP. Wang et al. (14) recently demonstrated in cardiomyocytes that mechanical strain suppressed TXNIP expression, and that increases in TRX activity followed. It has also been reported by Yoshioka et al. that TXNIP expression is decreased in pressure-overload cardiac hypertrophy followed by TRX-induced stimulation of cardiac cell growth (15). These results support the emerging concept that TXNIP is a critical regulator of biomechanical signaling in cardiovascular disorders. Therefore we hypothesized that steady laminar flow should inhibit TXNIP expression in ECs. Here we demonstrate a potentially novel mechanism for the atheroprotective effects of fluid shear stress via decreased 


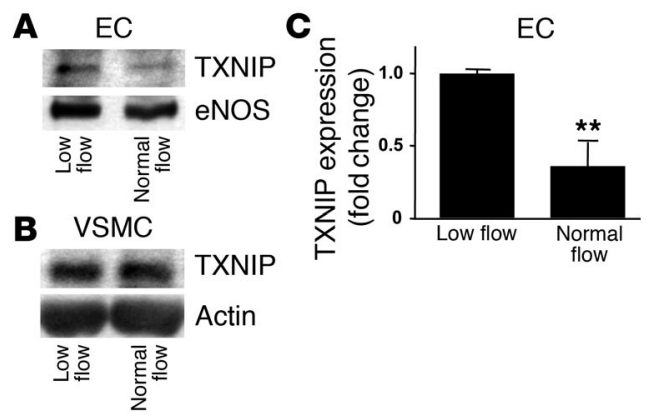

\section{Figure 1}

Normal flow downregulated TXNIP expression in ECs. After rabbit aortae were exposed to low flow $\left(0.4 \mathrm{dyn} / \mathrm{cm}^{2}\right)$ or normal flow $\left(12 \mathrm{dyn} / \mathrm{cm}^{2}\right)$ for 24 hours, EC (A) and VSMC (B) proteins were selectively purified, and immunoblotting was performed $(n=4-5)$. Equal protein loading was confirmed with eNOS or actin antibody. (C) TXNIP expression is shown as fold change relative to low flow. ${ }^{* *} P<0.01 \mathrm{vs}$. low flow.

TXNIP, increased TRX activity, and decreased activity of JNK, p38, and VCAM1 expression.

\section{Results}

Chronic flow downregulated TXNIP expression in rabbit aortic ECs. To examine the effects of chronic flow on TXNIP expression, rabbit aortae were exposed to low $\left(0.4 \mathrm{dyn} / \mathrm{cm}^{2}\right)$ or normal $\left(12 \mathrm{dyn} / \mathrm{cm}^{2}\right)$ fluid shear stress for 24 hours. EC and VSMC proteins were selectively purified from intact rabbit aorta as described previously (6). TXNIP expression in ECs was significantly inhibited by exposure to normal flow compared with low flow (64\% $\pm 17 \%$ inhibition; Figure 1, A and C; $P<0.01, n=5)$. Flow did not change TXNIP expression in VSMCs (Figure 1B; $n=4$ ).

Chronic flow increased TRX activity but not expression in rabbit aortic ECs. We next examined the effects of flow on TRX, which binds to TXNIP and is inhibited by TXNIP $(11,12)$. Exposure to normal flow for 24 hours significantly enhanced the insulin-reducing activity of TRX compared with exposure to low flow in ECs $(77 \% \pm 22 \%$ increase; Figure $2 \mathrm{~A} ; P<0.05, n=4)$. Expression of TRX protein in ECs did not change after flow (Figure $2 \mathrm{~B} ; n=5$ ).

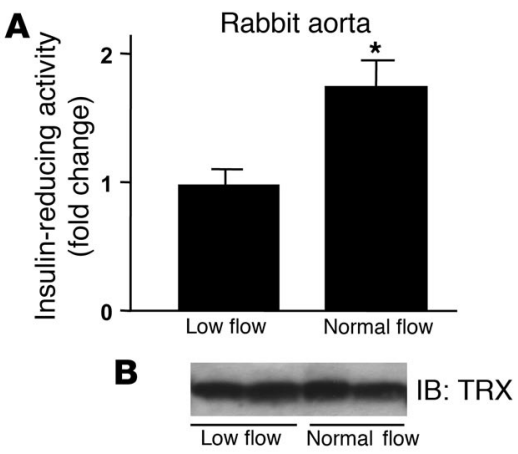

Figure 2

Normal flow increased the activity but not the expression of TRX in ECs. After rabbit aortae were exposed to low flow $\left(0.4 \mathrm{dyn} / \mathrm{cm}^{2}\right)$ or normal flow (12 dyn $\left./ \mathrm{cm}^{2}\right)$ for 24 hours, EC lysates were harvested. (A) TRX activity was determined by the insulin-reducing assay. Results are shown as fold change relative to low flow $(n=4)$. ${ }^{*} P<0.05$ vs. low flow. (B) TRX expression was unchanged in low and normal flow $(n=5)$.
Chronic flow downregulated TXNIP expression and upregulated TRX activity in human umbilical vein ECs. In human umbilical vein ECs (HUVECs), exposure to normal flow (fluid shear stress $=12 \mathrm{dyn} /$ $\mathrm{cm}^{2}$ ) for 24 hours decreased TXNIP expression compared with exposure to low flow $\left(0.4 \mathrm{dyn} / \mathrm{cm}^{2}\right)(50 \% \pm 15 \%$ inhibition; Figure $3 \mathrm{~A} ; P<0.05, n=4)$. While flow did not change TRX expression in HUVECs (Figure 3A; $n=4$ ), normal flow (12 dyn $/ \mathrm{cm}^{2}, 24$ hours) increased TRX activity $(40 \% \pm 8 \%$ increase; Figure $3 \mathrm{~B} ; P<0.05$, $n=4$ ), confirming our findings in rabbit aorta.

TXNIP small interfering RNA inbibited TNF activation of $p 38, \mathrm{JNK}$, and VCAM1 expression in HUVECs. We previously found that exposure to normal flow for 24 hours significantly inhibited TNF-stimulated JNK and p38 activity (6). TNF-induced VCAM1 expression in ECs was also significantly inhibited by exposure to normal flow (78\% inhibition) (6). It is logical to propose that normal flow prevents TNF-induced VCAM1 expression by regulating TXNIP-TRX-JNK/p38 pathways, since we previously showed that JNK and p38 mediated TNF-induced VCAM1 expression in rabbit aortic ECs, and it is known that TRX inhibits the ASK1JNK/p38 signaling pathway (8).

To show that inhibition of TXNIP contributes to the antiinflammatory effects of normal flow, we treated HUVECs with small interfering RNA (siRNA) against human TXNIP. TXNIP siRNA significantly decreased endogenous TXNIP protein expression $(53 \% \pm 11 \%$ decrease) compared with control siRNA without significant effect on eNOS (Figure 4; $P<0.01, n=6$ ). Compared with control siRNA, TXNIP siRNA significantly inhibited activation of $\mathrm{p} 38(48 \% \pm 12 \%$ inhibition) and JNK ( $42 \% \pm 10 \%$ inhibition) by TNF $(10 \mathrm{ng} / \mathrm{ml}, 15$ minutes; Figure 5 ; $P<0.05, n=3)$. In contrast, TNF activation of ERK1/2 (Figure $5 ; n=3$ ) and NF-KB (measured by IкB- $\alpha$ degradation; data not shown; $n=2$ ) was not inhibited, which suggests a specific role for TXNIP in TNF-mediated signal events related to $\mathrm{p} 38$ and JNK. TXNIP siRNA also significantly inhibited TNF-induced ( $10 \mathrm{ng} / \mathrm{ml}, 6$ hours) VCAM1 expression in HUVECs $(48 \% \pm 3 \%$ inhibition; Figure 6; $P<0.01, n=3)$. TNF-induced VCAM1 expression was almost completely blocked by inhibition of p38 (30 $\mu \mathrm{M} \mathrm{SB} 203580 ; n=4)$ but not by inhibition of ERK1/2 (30 $\mu \mathrm{M}$ PD98059; $n=3)$. Of note, the JNK inhibitor $(10 \mu \mathrm{M} \mathrm{SP} 600125 ; n=4)$ had no effect on VCAM1 expression in HUVECs, in contrast to our results in rabbit aorta (6). These
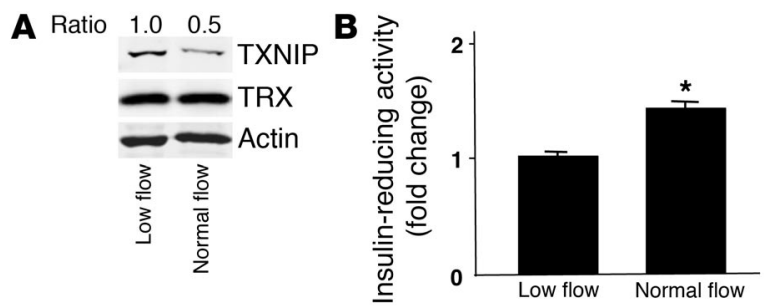

Figure 3

Normal flow downregulated TXNIP expression and upregulated TRX activity in HUVECs. After HUVECs were exposed to low flow (0.4 dyn $/ \mathrm{cm}^{2}$ ) or normal flow (12 dyn/ $\left./ \mathrm{cm}^{2}\right)$ for 24 hours, proteins were harvested. (A) TXNIP and TRX expression was determined by immunoblotting $(n=4)$. Equal protein loading was confirmed with actin antibody. TXNIP expression is shown as fold change relative to low flow. The ratio in normal flow differed significantly from that in low flow $(n=4, P<0.05)$. (B) TRX activity was determined by the insulin-reducing assay. Results are shown as fold change relative to low flow $(n=4)$. ${ }^{*} P<0.05$ vs. low flow. 
A

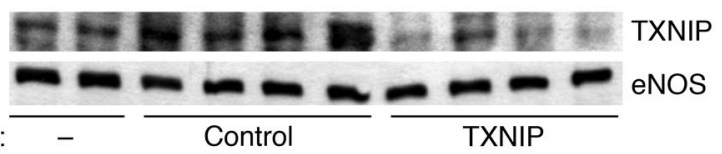

B

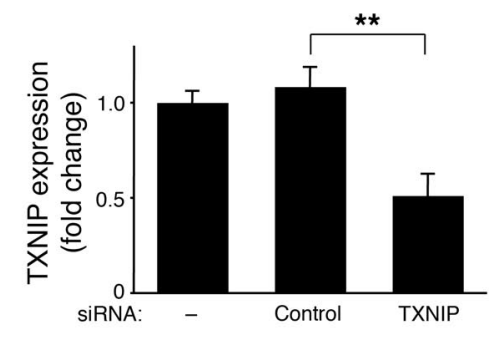

data suggest that inhibiting TXNIP decreases EC inflammatory responses (measured by TNF activation of VCAM1) by preventing p38 and/or JNK activation.

TXNIP siRNA increased TRX binding to ASK1 in HUVECs. Junn et al. (12) showed that binding of TRX to ASK1 was significantly reduced when TXNIP was overexpressed, which suggests that TXNIP can compete with TRX for binding to ASK1. Thus we examined the effects of decreasing TXNIP on interaction of TRX with ASK1. Treatment of HUVECs with TXNIP siRNA increased ASK1 binding to TRX (Figure 7A; $n=4$ ). TXNIP siRNA did not change the expression of ASK1 and TRX (Figure 7B). These results suggest that TXNIP regulates the binding of TRX to ASK1 in HUVECs.

TXNIP overexpression angmented TNF activation of $p 38$ and JNK in bovine aortic ECs. To further examine the contribution of TXNIP to inflammatory responses in ECs, we overexpressed TXNIP in bovine aortic ECs (BAECs). Overexpression of TXNIP significantly enhanced TNF-mediated activation of p38 (23\% $\pm 7 \%$ increase; Figure 8; $P<0.05, n=3)$ and JNK $(33 \% \pm 11 \%$ increase; Figure 8; $P<0.05, n=4)$, indicating an important role for TXNIP-TRX interactions in regulating p38 and JNK in ECs.

TNF-induced VCAM1 expression was decreased in TXNIP-deficient mice. We next examined the role of TXNIP in inflammatory responses in vivo. Recently, an animal model of combined hyperlipidemia (the HcB-19/Dem mouse), including hypertriglyceridemia, hypercholesterolemia, elevated plasma apoB, and increased triglyceride-rich lipoproteins, was shown to have a nonsense mutation in TXNIP, resulting in very low protein expression (16). It appears that the hyperlipidemia results from excess triglyceride and cholesterol synthesis by the liver rather than impaired EC function. Aortae from HcB-19 mice had nearly

\section{Figure 5}

TXNIP siRNA inhibited TNF activation of p38 and JNK but not ERK1/2 in HUVECs. After HUVECs were transfected with either control or TXNIP siRNA, TNF $(10 \mathrm{ng} / \mathrm{ml})$ was added for 15 minutes. MAPK activation was determined by immunoblotting using phosphospecific ( $p-$ ) antibody. Equal loading was confirmed with total MAPK antibodies. Representative blots from 3 independent experiments are shown. Quantitation of the ratio of phospho-MAPK in lysates from cells treated with control or TXNIP siRNA to phospho-MAPK in lysates from cells not treated with siRNA is shown below each panel (average of 3 determinations).

\section{Figure 4}

TXNIP protein expression was decreased by siRNA in HUVECs. HUVECs were transfected with either control or TXNIP siRNA. (A) TXNIP expression was determined by immunoblotting from 4-6 independent experiments. Equal protein loading was confirmed with eNOS antibody. (B) TXNIP expression is shown as fold change relative to no siRNA. ${ }^{*} P<0.01$ vs. control siRNA.

undetectable TXNIP (Figure 9; $n=2$ ), confirming the previously published data showing decreased TXNIP mRNA expression (16). In HcB-19 aorta, TNF-induced ( $15 \mathrm{ng} / \mathrm{ml}, 6$ hours) VCAM1 expression was suppressed (51\% decrease; Figure 9; $n=2)$. It appears that even under resting conditions VCAM expression in HcB-19 aorta is less than in wild-type aorta. These results support our hypothesis that inhibiting TXNIP expression contributes to the antiinflammatory effects of flow in ECs.

\section{Discussion}

The major findings of the present study are that physiologic fluid shear stress decreases TXNIP expression and limits proinflammatory events mediated by the TNF-ASK1-JNK/p38 pathway (Figure 10). We found in intact aortae that physiologic flow decreased TXNIP expression, and that decreased TXNIP expression was associated with decreased TNF-mediated VCAM1 expression. To our knowledge, the results are the first demonstration of the physiologic function of TXNIP in vascular endothelium. The data support the evolving concept that TXNIP and TRX are key components of biomechanical signal transduction and establish them as potentially novel regulators of TNF signaling and inflammation in the endothelium.

Several results suggest that TXNIP is a critical target for the antiinflammatory effects of steady laminar flow. TXNIP was shown to inhibit the catalytic activity of TRX $(11,12)$. This is important because only catalytically active (or reduced) TRX can bind to ASK1 and inhibit ASK1-dependent JNK and p38 activity (8). Our results show that chronic normal flow increased the catalytic activity of TRX (as measured by insulin reduction) in arterial ECs, confirming data in cultured ECs (17). Thus, we suggest that flow inhibition of TXNIP expression makes more reduced TRX 


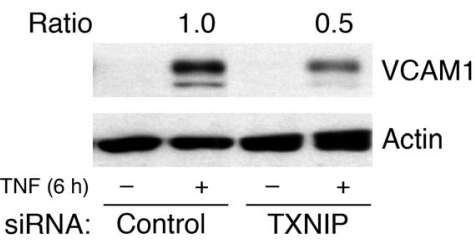

Figure 6

TXNIP siRNA inhibited TNF-induced VCAM1 expression in HUVECs. After HUVECs were transfected with either control or TXNIP siRNA, TNF- $\alpha(10 \mathrm{ng} / \mathrm{ml})$ was added for 6 hours. VCAM1 expression was determined by immunoblotting from 3 independent experiments. Equal protein loading was confirmed with actin antibody. VCAM1 expression is shown as fold change relative to control siRNA.

available to bind to ASK1 and inhibits JNK and p38 activation. We previously showed in cultured ECs that steady laminar flow of short duration (10 minutes) inhibited TNF activation of ASK1JNK pathways by increasing ASK1 association with its inhibitor 14-3-3 but not with TRX (5), which suggests that there are differences in regulation of ASK1 between acute and chronic flow. Another important mechanism by which flow inhibits inflammation is induction of a reduced intracellular redox state, since flow maintains the key antioxidant molecules TRX and glutaredoxin (18) in their reduced forms, which bind and inhibit ASK1. We previously found in cultured ECs that steady laminar flow inhibited $\mathrm{H}_{2} \mathrm{O}_{2}$-mediated activation of JNK by inducing a reduced intracellular redox state as measured by the level of reduced glutathione (19). Thus it seems likely that steady laminar flow limits EC inflammation by multiple regulatory mechanisms.

TXNIP was originally identified in HL-60 leukemia cells treated with 1,25-dihydroxyvitamin $\mathrm{D}_{3}(10)$. It was also shown that TGF- $\beta_{1}$ induced TXNIP in SNU-16/620 human stomach cancer cells (20). However, the stimuli that regulate TXNIP in cardiovascular tissues remain poorly defined. Wang et al. (14) recently demonstrated in cardiomyocytes that biomechanical strain suppressed TXNIP expression, and that increases in TRX activity followed. Overexpression of TXNIP sensitized cells to $\mathrm{H}_{2} \mathrm{O}_{2}$-induced apoptosis, whereas overexpression of TRX protected against injury. It was also demonstrated that PDGF suppressed TXNIP expression with increases in TRX activity and DNA synthesis in VSMCs (21). Conversely, overexpression of TXNIP abolished PDGF-induced TRX activity and DNA synthesis. These results suggest that TXNIP has proapoptotic effects in cardiomyocytes and VSMCs through the suppression of TRX activity. In the present study, we found in ECs that steady laminar flow decreased TXNIP expression and limited inflammation mediated by the TNF-ASK1-JNK/p38 pathways. Since inflammation and apoptosis are key mechanisms

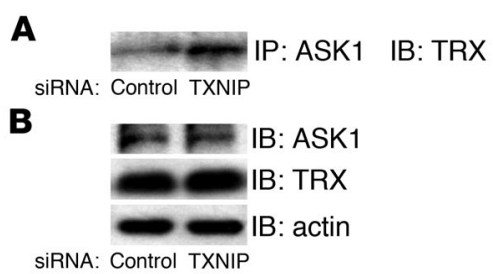

Figure 7

TXNIP siRNA increased TRX binding to ASK1 in HUVECs. (A) Interaction of ASK1 with TRX was examined by immunoblotting with TRX antibody after HUVEC lysates were immunoprecipitated with ASK1 antibody. (B) Equal loading was confirmed with ASK1, TRX, and actin antibodies. Results are representative of 4 independent experiments.

in atherosclerosis, we suggest that TXNIP is a potentially novel biomechanical effector of atherosclerosis.

\section{Methods}

Antibodies and reagents. Antibody sources were Santa Cruz Biotechnology Inc. for VCAM1, ERK1/2, p38, eNOS, actin, and IкB- $\alpha$; Cell Signaling Technology Inc. for phospho-ERK1/2, phospho-p38, JNK2, and ASK1; Promega Corp. for phospho-JNK; and American Diagnostica Inc. for human TRX1. Rabbit polyclonal antibody against mouse TXNIP and mouse $\mathrm{mAb}$ against human TXNIP were developed as previously described $(14,21)$. Reagent sources were Roche Applied Science for TNF- $\alpha$ and EMD Biosciences Inc. for the JNK inhibitor SP600125, the p38 inhibitor SB203580, and the ERK inhibitor PD98059.

Cell culture, siRNA oligonucleotide treatment, and plasmid transfection. HUVECs were purchased from Cascade Biologics Inc. and cultured in Medium 200 supplemented with low serum growth supplement (LSGS; Cascade Biologics Inc.). Cells at passages 3-5 were used for experiments.

To knock down TXNIP, we treated HUVECs with siRNA against human TXNIP coding region. The target sequences were 5'-AAGCCGTTAGGATCCTGGCTT-3' for human TXNIP and 5'-AATTCTCCGAACGTGTCACGT-3' for control nonsilencing siRNA (QIAGEN-Xeragon). HUVECs at greater than $90 \%$ confluence in $60-\mathrm{mm}$ dishes were used for transfection. For each dish, Lipofectamine 2000 (Invitrogen Corp.) was mixed with OptiMEM (Invitrogen Corp.; $200 \mu \mathrm{l}$ ), and then TXNIP or control siRNA (60 ng) was added to the solution, mixed gently, and incubated at room temperature for 20 minutes. This mixture was added to HUVECs in normal culture media $(2 \mathrm{ml})$, and cells were incubated for 1.5 hours. The medium was changed, and cells were recovered for 24 hours. The transfection was repeated, and after 24 hours, cells were used for experiments.

BAECs were purchased from Cambrex Corp. and were cultured in Medium 199 (Mediatech Inc.) supplemented with 10\% FetalClone III (HyClone), basal MEM vitamins, and amino acids (Invitrogen Corp.). Cells at passages 8-10 were used for experiments. BAECs at greater than $90 \%$ confluence

\section{Figure 8}

TXNIP overexpression augmented TNF-induced p38 and JNK activation in BAECs. BAECs were transfected with either control (pcDNA3.1) or pcDNA3.1-TXNIP and then treated with TNF- $\alpha(10 \mathrm{ng} / \mathrm{ml})$ for 15 minutes. Activation of p38 and JNK was determined by immunoblotting using phospho-specific antibody. Equal loading was confirmed with total MAPK antibodies. Representative blots from 3-4 independent experiments are shown.

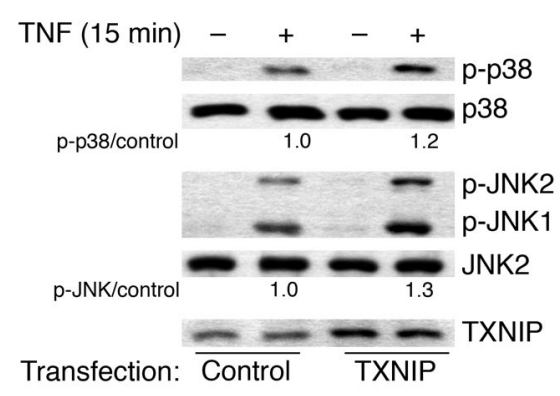




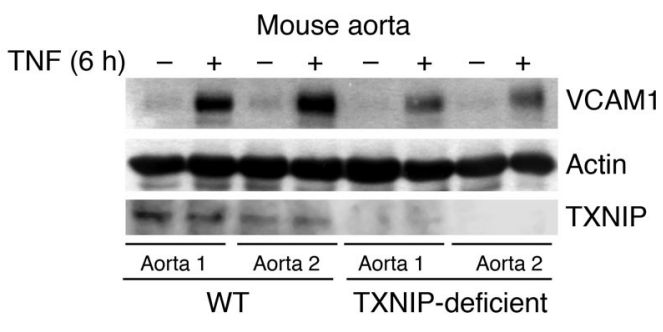

in 35- $\mathrm{mm}$ dishes were used for transfection. For each dish, Lipofectamine $2000(5 \mu \mathrm{l})$ was mixed with Opti-MEM $(250 \mu \mathrm{l})$, and then pcDNA3.1TXNIP $(14,21)$ or pcDNA3.1 $(2 \mu \mathrm{g})$ was added to the solution, mixed gently, and incubated at room temperature for 20 minutes. This mixture was added to BAECs in Opti-MEM ( $2 \mathrm{ml})$, and cells were incubated for 3 hours. The medium was changed, and cells were recovered for 24 hours. Cells were used for experiments after 18-24 hours of serum starvation.

For short-term exposure to flow, cells were cultured in 60-mm dishes and placed in a cone and plate viscometer as previously described (4). For long-term exposure to flow (24 hours), HUVECs in Medium 200 with LSGS were exposed to shear stress generated by a parallel-plate-type apparatus as previously described (22). Briefly, one side of the flow chamber had HUVECs growing to monolayers. The other side was a polycarbonate plate, and the 2 surfaces were held $200 \mu \mathrm{m}$ apart by a GlycoTech Corp. gasket. A closed circulation was arranged with a silicone tube and an air bag filled with medium to stabilize the flow. Medium was constantly circulated with a roller/tube pump at $37^{\circ} \mathrm{C}$ in $95 \%$ room air and $5 \% \mathrm{CO}_{2}$. Shear stress was calculated by the formula $6 \mu \mathrm{Q} / a^{2} b$, where $\mu$ is the viscosity of the perfusate (poise), $Q$ is flow volume $(\mathrm{ml} / \mathrm{s})$, and $a$ and $b$ are cross-section dimensions of the flow path $(\mathrm{cm})$.

Perfusion organ culture. Animal experiments were performed according to the guidelines of the NIH and the American Heart Association for the care and use of laboratory animals and were approved by the University of Rochester Animal Care Committee. Male New Zealand white rabbits (2-3 kg; Covance Research Products) were anesthetized with ketamine $(50 \mathrm{mg} / \mathrm{kg}$, i.v.) and xylazine $(2 \mathrm{mg} / \mathrm{kg}$, i.v.). Arterial segments from the descending thoracic aorta were isolated and cannulated at a constant pressure $(80 \mathrm{mmHg})(6,23,24)$. Isolated aortic segments were connected to a closed perfusion circuit consisting of a 3-port reservoir, a peristaltic pump, and a pressure chamber. Vessel segments were placed in a bath filled with culture medium identical to that used in the intraluminal compartment, consisting of serum-free DMEM containing antibiotics. To obtain a physiologic fluid viscosity ( 0.04 poise), $5 \%$ dextran (SigmaAldrich) was added. Previous reports found that this organ culture method preserved approximately $100 \% \mathrm{EC}$ and smooth muscle viability $(6,23$, 24). Flow rate was adjusted to $7.5 \mathrm{ml} / \mathrm{min}$ for low flow and $220 \mathrm{ml} / \mathrm{min}$

\section{Figure 10}

Flow regulates TXNIP in ECs. Chronic exposure to normal flow decreases TXNIP expression, and this results in increased TRX binding to ASK1. This inhibits cytokine activation of the JNK-p38 pathway and prevents proinflammatory events such as VCAM1 expression.

\section{Figure 9}

TNF-induced VCAM1 expression was decreased in TXNIP-deficient mouse aorta. After aortae from HcB-19 and control C3H mice were treated with TNF (15 ng/ml, 6 hours), vessel protein was harvested. Expression of VCAM1 and TXNIP was determined by immunoblotting in 2 aortic samples from 2 animals of each strain. Equal protein loading was confirmed with actin antibody.

for normal flow. In conditions of steady laminar flow, shear stress $(\sigma)$ is determined by flow rate $(Q)$, fluid viscosity $(\mu)$, and vessel diameter $(d)$ according to the relationship $\sigma=32 \mu Q / \pi d^{3}$. Based on this calculation, shear stress was $0.4 \mathrm{dyn} / \mathrm{cm}^{2}$ for low flow and $12 \mathrm{dyn} / \mathrm{cm}^{2}$ for normal flow. Organ culture of the aortic segments was carried out under sterile conditions in an incubator containing $5 \% \mathrm{CO}_{2}$ at $37.5^{\circ} \mathrm{C}$ for 24 hours.

Immunoprecipitation and immunoblotting. Immunoprecipitation and immunoblotting experiments were performed as described previously (4-6). The resulting autoradiograms were analyzed with NIH Image 1.60. Experiments were performed at least 3 times, and equal loading of protein was ensured by measurement of actin expression.

TRX-activity (insulin-reducing) assay. TRX activity was measured using the insulin disulfide reduction assay as described elsewhere with a slight modification $(12,14)$. Cell extracts $(10 \mu \mathrm{g}, 34 \mu \mathrm{l})$ were incubated at $37^{\circ} \mathrm{C}$ for 20 minutes with $1 \mu \mathrm{l}$ of reducing buffer composed of $50 \mathrm{mM}$ HEPES ( $\mathrm{pH}$ 7.6), $1 \mathrm{mM}$ EDTA, $1 \mathrm{mg} / \mathrm{ml} \mathrm{BSA}$, and $2 \mathrm{mM}$ DTT. Then $20 \mu \mathrm{l}$ of reaction buffer containing $200 \mu \mathrm{l}$ of $1 \mathrm{M}$ HEPES ( $\mathrm{pH} 7.6), 16 \mu \mathrm{l}$ of $0.5 \mathrm{M}$ EDTA, $80 \mu \mathrm{l}$ of $\operatorname{NADPH}(20 \mathrm{mg} / \mathrm{ml})$, and $500 \mu \mathrm{lof}$ insulin $(10 \mathrm{mg} / \mathrm{ml})$ was added. The reaction was started by the addition of $5 \mu$ l of bovine TRX reductase (American Diagnostica Inc.) and continued for 20 minutes at $37^{\circ} \mathrm{C}$. The reaction was terminated by the addition of $125 \mu \mathrm{l}$ of $10 \mathrm{M}$ guanidine- $\mathrm{HCl}$ and $1.7 \mathrm{mM}$ DTNB (3-carboxy-4-nitrophenyl disulfide), and the absorbance at $405 \mathrm{~nm}$ was measured spectroscopically.

TXNIP-deficient mouse. HcB-19 mice derived from the colony at UCLA (16, $25)$ were sacrificed immediately after delivery, and aortae were harvested. The HcB-19 mouse is a variant of the $\mathrm{C} 3 \mathrm{H}$ strain that was first described as a model of human familial combined hyperlipidemia (25). For the present study, age-matched ( 8 months) and strain-matched $\mathrm{C} 3 \mathrm{H} / \mathrm{HeJ}$ mice were used as controls. While the $\mathrm{C} 3 \mathrm{H} / \mathrm{HeJ}$ control is not genetically $100 \%$ identical to the HcB-19 mouse, these substrains are very similar. It is possible that subtle differences in TNF- and flow-mediated responses might alter the results shown here. Proteins were obtained by homogenization of aortae with Triton $\mathrm{X}$ lysis buffer after treatment with TNF- $\alpha$ for 6 hours at $37^{\circ} \mathrm{C}(6)$.

Statistical analysis. Data are shown as mean \pm SEM. Statistical evaluation was done by unpaired Student's $t$ test, and $P<0.05$ was taken as a significant difference. 


\section{Acknowledgments}

This study was supported by NIH grants HL62826 and HL64839 (to B.C. Berk) and HL64858 (to R.T. Lee). We are grateful to Y. Hojo, A. Mohan, M.B. Taubman, and Center for Cardiovascular Research members for useful suggestions. We also acknowledge the generosity of A.J. Lusis (UCLA, Los Angeles, California, USA) for providing $\mathrm{HcB}-19$ mice.
Received for publication August 10, 2004, and accepted in revised form December 12, 2004.

Address correspondence to: Bradford C. Berk, Center for Cardiovascular Research, Box 679, 601 Elmwood Avenue, University of Rochester, Rochester, New York 14642, USA. Phone: (585) 273-1946; Fax: (585) 273-1497; E-mail: bradford_berk@urmc.rochester.edu.
1. Gimbrone, M.A., Jr., Topper, J.N., Nagel, T., Anderson, K.R., and Garcia-Cardena, G. 2000 Endothelial dysfunction, hemodynamic forces, and atherogenesis. Ann. N. Y. Acad. Sci. 902:230-239.

2. Traub, O., and Berk, B.C. 1998. Laminar shear stress: mechanisms by which endothelial cells transduce an atheroprotective force. Arterioscler. Thromb. Vasc. Biol. 18:677-685.

3. Ross, R. 1999. Atherosclerosis: an inflammatory disease. N. Engl. J. Med. 340:115-126.

4. Surapisitchat, J., et al. 2001. Fluid shear stress inhibits TNF-alpha activation of JNK but not ERK1/2 or p38 in human umbilical vein endothelial cells: inhibitory crosstalk among MAPK family members. Proc. Natl. Acad. Sci. U. S. A. 98:6476-6481.

5. Liu, Y., Yin, G., Surapisitchat, J., Berk, B.C., and Min, W. 2001. Laminar flow inhibits TNF-induced ASK1 activation by preventing dissociation of ASK1 from its inhibitor 14-3-3. J. Clin. Invest. 107:917-923.

6. Yamawaki, H., Lehoux, S., and Berk, B.C. 2003. Chronic physiological shear stress inhibits tumor necrosis factor-induced proinflammatory responses in rabbit aorta perfused ex vivo. Circulation. 108:1619-1625.

7. Ichijo, H., et al. 1997. Induction of apoptosis by ASK1, a mammalian MAPKKK that activates SAPK/JNK and p38 signaling pathways. Science. 275:90-94

8. Saitoh, M., et al. 1998. Mammalian thioredoxin is a direct inhibitor of apoptosis signal- regulating kinase (ASK) 1. EMBO J. 17:2596-2606.

9. Yamawaki, H., Haendeler, J., and Berk, B.C. 2003 Thioredoxin: a key regulator of cardiovascular homeostasis. Circ. Res. 93:1029-1033.

10. Chen, K.S and DeLuca, H.F. 1994. Isolation and characterization of a novel cDNA from HL-60 cells treated with 1,25-dihydroxyvitamin D-3. Biochim. Biophys. Acta. 1219:26-32.

11. Nishiyama, A., et al. 1999. Identification of thioredoxin-binding protein-2/vitamin $\mathrm{D}(3)$ up-regulated protein 1 as a negative regulator of thioredoxin function and expression. J. Biol. Chem. 274:21645-21650.

12. Junn, E., et al. 2000. Vitamin D3 up-regulated protein 1 mediates oxidative stress via suppressing the thioredoxin function. J. Immunol. 164:6287-6295.

13. Schulze, P.C., et al. 2004. Hyperglycemia promotes oxidative stress through inhibition of thioredoxin function by thioredoxin-interacting protein. J. Biol. Chem. 279:30369-30374.

14. Wang, Y., De Keulenaer, G.W., and Lee, R.T. 2002. Vitamin D(3)-up-regulated protein-1 is a stressresponsive gene that regulates cardiomyocyte viability through interaction with thioredoxin. J. Biol. Chem. 277:26496-26500.

15. Yoshioka, J., et al. 2004. Thioredoxin-interacting protein controls cardiac hypertrophy through regulation of thioredoxin activity. Circulation. 109:2581-2586.

16. Bodnar, J.S., et al. 2002. Positional cloning of the combined hyperlipidemia gene Hyplip1. Nat. Genet. 30:110-116.

17. Hoffmann, J., Dimmeler, S., and Haendeler, J. 2003. Shear stress increases the amount of S-nitrosylated molecules in endothelial cells: important role for signal transduction. FEBS Lett. 551:153-158.

18. Song, J.J., et al. 2002. Role of glutaredoxin in meta- bolic oxidative stress. Glutaredoxin as a sensor of oxidative stress mediated by $\mathrm{H} 2 \mathrm{O} 2$. J. Biol. Chem. 277:46566-46575.

19. Hojo, Y., et al. 2002. Fluid shear stress attenuates hydrogen peroxide-induced c-Jun NH2-terminal kinase activation via a glutathione reductase-mediated mechanism. Circ. Res. 91:712-718.

20. Han, S.H., et al. 2003. VDUP1 upregulated by TGF-beta1 and 1,25-dihydroxyvitamin D3 inhibits tumor cell growth by blocking cell-cycle progression. Oncogene. 22:4035-4046.

21. Schulze, P.C., De Keulenaer, G.W., Yoshioka, J., Kassik, K.A., and Lee, R.T. 2002. Vitamin D3-upregulated protein-1 (VDUP-1) regulates redox-dependent vascular smooth muscle cell proliferation through interaction with thioredoxin. Circ. Res. 91:689-695.

22. Pi, X., Yan, C., and Berk, B.C. 2004. Big mitogenactivated protein kinase (BMK1)/ERK5 protects endothelial cells from apoptosis. Circ. Res. 94:362-369.

23. Bardy, N., Karillon, G.J., Merval, R., Samuel, J.L., and Tedgui, A. 1995. Differential effects of pressure and flow on DNA and protein synthesis and on fibronectin expression by arteries in a novel organ culture system. Circ. Res. 77:684-694.

24. Lehoux, S., Esposito, B., Merval, R., Loufrani, L., and Tedgui, A. 2000. Pulsatile stretch-induced extracellular signal-regulated kinase $1 / 2$ activation in organ culture of rabbit aorta involves reactive oxygen species. Arterioscler. Thromb. Vasc. Biol. 20:2366-2372.

25. Castellani, L.W., et al. 1998. Mapping a gene for combined hyperlipidaemia in a mutant mouse strain. Nat. Genet. 18:374-377. 